\title{
Un análisis crítico al modelo aprendizaje servicio: Su implementación en la Universidad Católica de Temuco
}

\author{
A critical analysis to the Service-Learning Model: \\ Its implementation in the Catholic University of Temuco
Luis Vivero Arriagada ${ }^{a}$, Walter Molina Chávez $^{\mathrm{b}}$ \& Danitza Standen Silva ${ }^{\mathrm{c}}$
a Universidad Católica de Temuco, Chile. $\triangle$ luisvive@gmail.com [orcid.org/0000-0002-6459-1386]
b Universidad de Magallanes, Chile. walter.molina@umag.cl [orcid.org/d.org/0000-0001-6497-0258]
c Universidad Católica de Temuco, Chile.danitza.standen@gmail.com \\ [orcid.org/0000-0001-5369-8092]
}

\section{RESUMEN}

El objetivo del presente trabajo es identificar los elementos fundamentales del Modelo Aprendizaje Servicio (MAS) y su implementación en el proceso educativo universitario. Se sustenta desde una metodología cualitativa, sobre la base de una revisión y análisis documental en relación a definiciones teóricas y conceptuales del modelo. Esto se articula con la experiencia en la Universidad Católica de Temuco, que, asumiendo los cambios que ha experimentado la sociedad contemporánea, se ha comprometido en desarrollar nuevas formas de enseñanza-aprendizaje y, en consecuencia, incorpora en su proyecto educativo la formación basada en competencias. Como conclusión se sostiene que el MAS no solo permite que los estudiantes desarrollen sus habilidades y destrezas técnicas, sino que además posibilita una comprensión crítica de la realidad y una articulación de los conocimientos de diversos ámbitos disciplinares aprendidos en la sala de clases con otros saberes, modos de hacer y formas de aprender.

PALABRAS CLAVE: aprendizaje a través de la experiencia, educación basada en las competencias, competencia profesional, competencias para la vida. 


\section{Vivero et al.}

\section{ABSTRACT}

This work aims at identifying key elements of the Service-Learning Model (MAS) and its implementation at the university educative process. It is based on a qualitative methodology, founded on a review and documentary analysis related to theoretical and conceptual definitions of the model. This study links with the context of the Catholic University of Temuco which, assuming the changes in modern society, has committed to develop new ways of teaching-learning and, consequently, incorporates to its project the competencebased education. Finally, it is argued that MAS does not only allow students to develop their technical skills and abilities, it also enables a critical understanding of reality and an interaction of various disciplinary fields learned in the classroom with other knowledge, ways of doing and ways of learning.

KEY WORDS: experience-based learning, competences-based education, professional competence, life competencies.

\section{INTRODUCCIÓN}

Los discursos del paradigma dominante, adornados en las consignas del modelo por competencias, plantean que los procesos de formación profesional debieran impactar en el aprendizaje y en la formación de habilidades específicas y genéricas de los/as estudiantes. Los partidarios de este emergente modelo lo consideran como un nuevo paradigma educativo que puede hacer más efectivo el proceso de enseñanza-aprendizaje (Tobón, 2008; Asún et al. 2013). Sin duda, este enfoque implica cambios y transformaciones profundas en los diferentes niveles educativos, y, de acuerdo con sus seguidores, conlleva desafíos inherentes para los diferentes actores del campo educativo universitario. Algunos de ellos se relacionan con la necesidad de comprometerse con una docencia de calidad, basada en el desarrollo de capacidades o competencias profesionales, tanto genéricas como específicas, en diversos contextos disciplinarios y de aprendizaje significativo para los/as estudiantes. De acuerdo con algunos autores (Tobón, 2008), este modelo busca articular multidimensionalmente el desarrollo de las competencias profesionales en el dominio cognitivo, procedimental y actitudinal. Desde una perspectiva analítica más global, se relaciona este nuevo enfoque formativo educativo con ciertos planteamientos del paradigma del pensamiento complejo (Morin, 2006; Tobón, 2008).

En este contexto analítico amplio, y desde este enfoque formativo se sostiene que, una vez terminada una carrera universitaria, el/a estudiante egresado haya obtenido las herramientas, capacidades o competencias profesionales las cuales le permitan aportar a la sociedad con una serie de destrezas, conocimientos, valores y actitudes explicitadas en sus respectivos perfiles de egreso. Dado lo anterior, se entendería que las prácticas pedagógicas actualmente en uso en los actuales planteles universitarios no solo deben entregar conocimientos, sino que 
deben aportar a la formación de competencias en los/as estudiantes, que les permitan resolver problemas en escenarios de alta complejidad y renovación de los saberes (Jouannet et al. 2013).

En consecuencia, y siguiendo esta lógica del modelo formativo basado en competencias, el gran desafío del docente universitario es cómo lograr que las y los jóvenes, además del contexto inherente asociado a su área de formación profesional o disciplinaria específica, vivencien experiencias reales de "formación práctica" en su vida universitaria (Ugueño, 2015), y situándose tanto en ámbitos sociales próximos como en una multiplicidad de entornos que ofrece el mundo globalizado. Esta participación activa y real en la comunidad es una experiencia formativa irrenunciable para una completa educación en valores y para la ciudadanía en pleno siglo XXI. En este contexto de la necesidad de una respuesta dinámica a los actuales desafíos y demandas formativas que las sociedades contemporáneas les hacen a los sistemas universitarios en el mundo contemporáneo, estos últimos han ido incorporando, paulatinamente, en el campo de la educación superior un tipo de aprendizaje a través de la experiencia conocido como Modelo Aprendizaje Servicio (en adelante MAS).

Desde una perspectiva teórica amplia, existen variadas definiciones formales para el MAS, no obstante, en Chile, este concepto es conocido como un enfoque pedagógico de enseñanza-aprendizaje (Barrios Araya et al. 2012). Al respecto, estos autores plantean que dicho modelo de aprendizaje puede ser aplicado en cursos extensos, prácticas y tesis; así como también incorporado dentro de los programas más amplios de vinculación con el medio, en donde las Instituciones de Educación Superior (IES) y sus estudiantes contribuyen a resolver problemáticas sociales objetivables mediante un servicio de calidad, por medio del cual y de manera integrada y colaborativa los tres actores presentes en el proceso de formación: docentes, estudiantes y agentes socios comunitarios, se vinculan y trabajan en conjunto en pos de resolver problemáticas que afectan generalmente a toda una comunidad (Facultad de Ciencias Físicas y Matemáticas, Universidad de Chile, 2019). Así entonces, el MAS vendría a contribuir a resolver aquellas problemáticas que se presentan en el contexto social específico, sin que éstas hayan sido previamente problematizadas o conceptualizadas participativamente a partir de un análisis histórico-político, cultural y económico de los actores sociales implicados en la configuración de dichos procesos. Esta no incorporación de los actores de base, situados en el territorio, emerge como una debilidad del MAS dado que estaría atacando unilateralmente el síntoma de un fenómeno mucho más complejo y multidimensional.

En relación a lo anterior, no podemos pasar por alto que desde fines del siglo XX y lo que va transcurrido del siglo XXI se han producido y consolidado profundos cambios en el contexto social, político, económico, tecnológico y cultural. Esto, sin duda, genera nuevas demandas en el campo de la educación superior, tanto a nivel nacional como internacional, y en el contexto latinoamericano se tematizan como exigencia de un acceso inclusivo, apertura a la interculturalidad y un carácter no sexista de los servicios educativos de nivel superior, de tal modo que rompan con las brechas de desigualdad e injusticia social (Atria, 2013, 2014; 
Bellei, 2015). Pero, por otro lado, el paradigma educativo dominante ha ido consolidando la educación como una mercancía y, por lo mismo, los enfoques en relación a la producción de capital humano se han ido configurando en torno a la consolidación de un mercado educativo en Chile, en el cual hay oferentes y demandantes de servicios educativos y éstos tienden a (des)alinearse con demandas funcionales de los mercados ocupacionales y del trabajo (Atria, 2013, 2015; Bellei, 2015). Esto se materializa en el campo de la educación superior chilena, latinoamericana y a nivel mundial, de una creciente tendencia de las Instituciones de Educación Superior (IES) de privilegiar el paradigma formativo que asume como relevante e innovador un modelo de Aprendizaje Basado en Competencias (Villa \& Poblete, 2007). Nos parece que, de acuerdo a estos autores, el nuevo enfoque resalta la importancia de la vinculación de la formación académica con el mundo laboral y profesional, de modo que el estudiantado sea capaz de aplicar el conocimiento adquirido y las competencias desarrolladas en su proceso formativo para poder desempeñar puestos laborales con eficacia y en el marco de una lógica productivista y funcional a los intereses de acumulación de capital.

En consecuencia, la estrategia de aprendizaje representa un recurso relevante para lograr los objetivos de aprendizaje significativo, como se plantea en el modelo por competencias, y, a la vez, conseguir una contribución de las y los jóvenes al logro del bien común y la adquisición de valores y virtudes cívicas (Puig Rovira et al. 2011). En este sentido, lo que nos llama la atención es la mirada descontextualizada que se tiene del aporte del estudiantado universitario, lo cual limita el aporte potencial que pudiesen realizar las y los estudiantes en las áreas en las cuales se insertan laboralmente, más aún, cuando esos espacios pudieran estar atravesados por condiciones de desigualdad y exclusión social, lo cual no es integrado adecuadamente a sus procesos formativos. Por lo tanto, consideramos que tanto el Modelo por Competencias y MAS, al no partir de una problematización situada del contexto histórico-político, tanto en la instancia local como global de los fenómenos abordados, podrían contribuir a la invisibilización de aquellas condiciones estructurales que producen y reproducen la desigualdad y exclusión social en las sociedades contemporáneas. Esto, incluso, en las propias realidades y vivencias cotidianas de los/as estudiantes que provienen de sectores excluidos y que en el último quinquenio han aumentado en los planteles universitarios chilenos, a partir de las políticas públicas educativas no contributivas que han implementado mecanismos de gratuidad y eliminación de los pagos de los aranceles universitarios para los/as estudiantes pertenecientes a los quintiles de ingresos más bajos de la población chilena actual.

En coherencia con lo anterior, este artículo tiene una doble finalidad. Por un lado, identificar los elementos fundamentales del MAS y su implementación en la Universidad Católica de Temuco (en adelante UCTemuco). Esto parte de la comprensión de la profesión como vocación de servicio, lo cual ha significado incorporar en los itinerarios formativos actividades curriculares de servicio socio-comunitario, a fin de generar un aprendizaje significativo, contextualizado, y proporcionar soluciones a las necesidades del entorno y 
sus actores sociales específicos. Por otro lado, a partir de estos elementos objetivados en los documentos analizados, concretamente los Proyectos Educativos Institucionales (PEI) y la inserción del MAS en ellos, nos proponemos levantar un análisis crítico en torno a este modelo formativo. Consideramos que, sin una articulación con el análisis de las condiciones estructurales del modelo de sociedad actual, la implementación del MAS no sería más que una respuesta parcial, instrumental y descontextualizada, a las problemáticas históricas y socioestructurales que ha generado el paradigma productivista que encarna el neoliberalismo en su implementación en la realidad latinoamericana y especialmente en Chile en las últimas cuatro décadas.

\section{CONSIDERACIONES METODOLÓGICAS}

En términos metodológicos el proceso investigativo, que da origen al presente artículo, fue de tipo cualitativo (Canales, 2006, 2013a, 2013b). Esto por cuanto se busca identificar e interpretar críticamente los ejes estructurantes en la producción del conocimiento respecto del modelo MAS que se ha implementado en el caso de una universidad confesional, como lo es la UCTemuco. Es decir, se trata de una investigación que se inscribe en una revisión y análisis documental, por lo tanto, la unidad de análisis es el discurso institucional contenido en el corpus documental analizado. En este proceso se generó una matriz de análisis, lo cual permitió ordenar el contenido de los textos revisados (Van Dijk, 1989). Para lo anterior, tomando como referencia la propuesta de Van Dijk (1989) se consideraron las macro-reglas que propone el autor para el análisis de textos: i) supresión, ii) generalización, iii) construcción o integración. La aplicación de estas reglas formales de análisis permite seleccionar las proposiciones que son textualmente pertinentes, es decir, que desempeñen un papel relevante en la interpretación de las otras oraciones de un determinado texto (Van Dijk, 1996). Esta modalidad de análisis permitió un ordenamiento del discurso para su posterior descripción y análisis interpretativo de los documentos relativos tanto al proyecto educativo de la UCTemuco como en relación al MAS, recuperando por esta vía analítica los temas o macro-estructuras de los textos y discursos que describen y enuncian este modelo de aprendizaje.

\section{MODELO APRENDIZAJE SERVICIO: EL CASO DE LA UNIVERSIDAD CATÓLICA DE TEMUCO}

En el informe de la UNESCO (1997) se sostiene que la educación a lo largo de la vida de una persona se basa en cuatro pilares que surgen de las necesidades del ser humano: aprender a ser, aprender a conocer, aprender a hacer y aprender a vivir juntos. Posteriormente, los modelos formativos en su mayoría, tanto para la educación escolar como para la educación universitaria, se apoyaron en estas cuatro dimensiones axiológicas de los procesos educativos para formular sus propios enfoques, dominios 
y fines educacionales; incluido los emergentes enfoques de Aprendizaje Basado en Competencias que hegemonizaron el discurso del diseño curricular, didáctico y evaluativo en la educación superior chilena a partir de inicios del siglo XXI. Para que se concrete la agenda educativa y los objetivos de desarrollo humano definidos por los organismos educativos internacionales es necesario redefinir su finalidad, explicitar y precisar los valores universales que proclama y promueve, y asumir un categórico compromiso por la mejora y el desarrollo social (Aguilar \& Bize, 2000). Desde este marco analítico, la educación, escolar y terciaria, tiene la responsabilidad de responder a la necesidad común y constante para todas las personas: el desarrollo de habilidades y capacidades personales, intelectuales y espirituales que necesita cada estudiante para avanzar en el ambiente que lo rodea y en el mundo actual (Lepeley, 2001).

Desde una perspectiva crítica, sostenemos que todo modelo de educación contiene tres preguntas cuyas respuestas definen en buena medida su contenido y orientación. La primera pregunta es el ¿Para qué del modelo?, la respuesta a esta interrogante determina el contenido de su dimensión política e ideológica. La segunda pregunta es el ¿Qué conocimientos se deben aprender-enseñar a partir de este modelo?, su respuesta define el contenido epistemológico del modelo de educación. Una tercera, y no necesariamente la última, pregunta es el ¿Cómo orientar el modelo?, es decir, la dimensión metodológica del modelo, y que implica la forma concreta de su implementación.

En relación a nuestro caso de estudio, la UCTemuco se declara como una institución de servicio y bien público, considerando su triple condición de universidad, católica y regional (Universidad Católica de Temuco, 2016). En el marco de su proceso de mejora continua ha implementado un modelo de enseñanza basado en tres saberes del aprendizaje, ser, saber ser y saber hacer, modelo que propende la dotación de herramientas que facilitan la formación de personas íntegras, para los campos donde intervienen (Universidad Católica de Temuco, 2019). Una de las herramientas pedagógicas que mejor refleja el sentido del modelo de enseñanza adoptado en esta universidad, pareciera ser el Aprendizaje Servicio (en adelante AS), mediante el cual las/os estudiantes lograrían un mejor y eficaz acercamiento a la realidad social y a los actores que la componen (Barrios Araya et al. 2012; Ugueño, 2015; Santander, 2017).

De acuerdo a lo sostenido por Ugueño (2015), a partir del año 2009 la UCTemuco articula un nuevo Modelo Educativo institucional, orientado a adecuar la formación universitaria para responder, tanto a las nuevas demandas de la formación disciplinaria/profesional como al mundo del trabajo al que deben insertarse sus egresados. En esta perspectiva, la Universidad define cinco ejes para su nuevo modelo educativo: "Estos son 1) Centralidad del proceso de aprendizaje, 2) Formación basada en competencias como herramienta para el aseguramiento de la calidad con foco en la integración de éstas en todos los planes de estudio, 3) TICs para el apoyo de los perfiles de egreso, 4) Educación para toda la vida, en un continuo articulado, y 5) Formación humanista cristiana" (Ugueño, 2015, p. 248). 
Como se puede observar, este modelo de enseñanza le ha permitido a la UCTemuco posicionarse como institución de formación profesional y técnica en sintonía con la realidad de la macro-zona sur de Chile y con una sociedad del conocimiento que se actualiza constantemente, en donde el saber ser se convierte en una fase importante dentro del quehacer universitario, pues forma profesionales idóneos, conforme a la realidad circundante al contexto regional y nacional, articulando acciones con aquellos actores sociales de relevancia en el país, donde son los propios estudiantes quienes, trabajando en conjunto con la comunidad, abordan una diversidad de temáticas relevantes que son de índole social y comunitaria y que a la vez van en beneficio directo de sus propias necesidades.

En este sentido, la aplicación del MAS en la UCTemuco permite que sus estudiantes inicien tempranamente en su formación de pregrado, acciones que aportan servicios de algún tipo que contribuyen en alguna dimensión al desarrollo integral de las comunidades y que fortalecen las acciones que, desde las propias políticas públicas, van dirigidas a la solución de las problemáticas sociales locales. De este modo, la Universidad se posiciona como una red de apoyo fundamental a los procesos de mejora social de las comunidades locales, generando espacios de intervención para la ejecución de intervenciones más profundas, desde los otros entes directamente involucrados en los procesos de desarrollo local (Aguilar \& Bize, 2000). Por su parte, al sintetizar las características básicas del MAS y su aplicación en la UCTemuco, Ugueño (2015) señala que son las siguientes: 1) Responden a una necesidad comunitaria genuina, 2) Integran objetivos curriculares y de servicio, 3) Asignan tiempo estructurado de reflexión de la relación entre contenidos y servicio, 4) Fomentan el protagonismo de los estudiantes, 5) Promueven una sociedad entre comunidad y universidad.

Lo anterior ha permitido a la Universidad contribuir a la formación de ciudadanas/os técnicamente capacitados, empáticos, responsables, conscientes y solidarios, que evidencian una serie de valores capaces de impregnar en la sociedad y responden a las necesidades de ésta desde sus respectivas disciplinas, validando la pedagogía del MAS como herramienta de aprendizaje vivencial. Esto, pone a la/el estudiante al centro de los procesos de enseñanzaaprendizaje y obliga, para estos fines, su vinculación con la comunidad, pudiendo éstos, desde el pregrado, ser un aporte a otros individuos de la sociedad, grupos y comunidades, en un trabajo que genera por un lado el aprendizaje y por otro lado el beneficio social. Esta perspectiva podría fortalecerse como proyecto político-pedagógico, como un proceso liberador, entendiendo que "la educación liberadora, problematizadora, ya no puede ser un acto de depositar, de narrar, de transferir o trasmitir $<$ conocimientos $>$ y valores a los educandos, meros pacientes, como lo hace la educación <bancaria >, sino un acto cognoscente" (Freire, 2006, p. 84). Esto, sin duda, es un desafío y un debate que necesariamente consideramos debería darse antes de la implementación de una propuesta de AS, de lo contrario esto queda solo en un nivel instrumental, y sus logros y aprendizajes se limitarían a este nivel, y no desde una problematización crítica y dialéctica de los/as educandos con su contexto histórico social. 


\section{IMPORTANCIA DEL APRENDIZAJE SERVICIO EN LA UNIVERSIDAD CATÓLICA DE TEMUCO Y SU ESTRATEGIA DE GESTIÓN}

El AS implica ejecutar actividades que combinan el servicio a la comunidad con el aprendizaje reflexivo de conocimientos, habilidades y valores. Nos preocupa que esa idea de "servicio a la comunidad" venga impregnada de una idea asistencialista y mesiánica, por cuanto ello "es una forma de acción que roba al hombre condiciones para el logro de una de las necesidades fundamentales de su alma- la responsabilidad” (Freire, 2004, p. 50). Esta preocupación tiene sentido en cuanto algunos autores señalan que es conocido el impacto formativo y transformador del voluntariado y las acciones solidarias, que contribuyen a la comprensión compleja de la realidad y a la formación de actitudes y valores personales para la transformación social (Puig Rovira et al. 2011).

La novedad y la riqueza del AS residen en la integración de dos elementos -servicio a la comunidad y aprendizaje significativo- en un solo proyecto coherente y bien articulado que potencia la capacidad formativa de ambos elementos. Por una parte, la acción de servicio transforma y da sentido a los aprendizajes y, por la otra, el desarrollo de un aprendizaje activo y significativo mejora la acción de solidaridad. Elementos que, además, permiten la formación de competencias reflexivas y críticas, fomentan el desarrollo de un compromiso solidario y facilitan el ejercicio responsable de la ciudadanía (Puig Rovira et al. 2011); generando, finalmente, capital social en los estudiantes, lo que les hace superar el "yo" para situarse en el "nosotros", abandonando el individualismo (White \& Henry, 1999). En esta línea nos parece relevante, al menos, poner en tensión esta mirada un tanto mesiánica, en cuanto que “(...) voluntarismo y espontaneísmo tienen ambos su falsedad en el menosprecio de los límites. El primero no respeta los límites porque para él solo hay uno, el de la voluntad del voluntarista. En el segundo el intelectual no interviene, no orienta, se cruza de brazo" (Freire, 1996, p. 51). Aquí es donde nos parece que podría estar el riesgo de un modelo como éste, al no problematizar esa acción concreta de colaboración que se desarrolla en los espacios en que pone en curso las competencias profesionales.

Una de las principales características que poseen las Instituciones de Educación Superior (IES), hoy en día, es que deben mirar la formación desde un prisma que articule la teoría y práctica y que esto genere una relación más estrecha entre universidad y sociedad (Unesco, 1997). En esta perspectiva el MAS es una metodología que representa de mejor manera la relación entre la universidad y su entorno y supone la integración de los objetivos pedagógicos y de servicio, siendo tres los elementos centrales: 1) el aprendizaje, 2) el servicio y 3) la reflexión estructurada (Hansen, 2012). Como parte del proceso de implementación del aprendizaje servicio en las universidades chilenas, surge la Red Nacional de Aprendizaje Servicio (REASE) que define al AS de la siguiente forma:

Enfoque pedagógico de enseñanza-aprendizaje aplicado en cursos, prácticas y tesis, en donde se resuelven problemáticas sociales reales mediante un servicio de calidad, en el 
que de manera integrada y colaborativa los tres actores presentes en el proceso: docentes, estudiantes y socios comunitarios, se vinculan y trabajan en conjunto (Red de Aprendizaje y Servicio, Chile, 2019).

Otra definición establece que es una metodología de enseñanza y aprendizaje mediante la cual los jóvenes desarrollan sus conocimientos y competencias a través de una práctica de servicio a la comunidad (Tapia, 2008). Una tercera definición, propuesta por el Centro Promotor de Aprendizaje y Servicio de Barcelona, lo explica como una propuesta educativa que combina procesos de aprendizaje y de servicio a la comunidad en un solo proyecto bien articulado donde los participantes aprenden al trabajar en necesidades reales del entorno con la finalidad de mejorarlo (Centro Promotor de Aprendizaje Servicio, 2019).

La reflexión en AS toma sentido antes, durante y después de la experiencia de servicio, y busca que los/as estudiantes y docentes ajusten procedimientos metodológicos en base a las necesidades de la comunidad (Cooper et al. 2013). El AS favorece el trabajo con personas de diferentes realidades socio-culturales, desarrollando en los/as estudiantes un entendimiento interpersonal, habilidades para trabajar productivamente con otros, apreciación de sistemas valóricos opuestos, apreciación sobre diferencias culturales, consideración por otros, y la habilidad de preocuparse y cuidar de otros. Así también, otras definiciones conciben al AS como una experiencia de aprendizaje a través de la solidaridad (Espinoza \& Rodríguez de la Piedra, 2015). Esto lo confirma Eyler (2001), en cuanto a que los espacios de reflexión le dan la posibilidad a los/as estudiantes de significar su práctica en la comunidad, relacionar los contenidos del curso con la experiencia de servicio, formular preguntas, proponer teorías y planes de acción y expresar sus ideas, lo que es crítico para la consolidación de aprendizajes. Esto, en un sentido freiriano, debe darse por medio de un proceso reflexivo históricocrítico, en donde los/as educandos se reconozcan desde su propia historicidad, y no que se transforme en una experiencia irreflexiva y voluntarista, que no haga sino reproducir las formas de dominación impuestas por las elites.

Los proyectos de AS implican la incorporación de metodologías innovadoras que supongan algún cambio en relación con otras estrategias de aprendizaje (Puig Rovira et al. 2011). El aprendizaje servicio se ha incorporado al currículo de algunas universidades chilenas, a lo que paulatinamente se irán sumando más entidades de educación superior, continuando con la dinámica de lo que ha sido el fenómeno en Latinoamérica y el resto del mundo (Centro Latinoamericano de Aprendizaje y Servicio Solidario, 2019).

Destacando lo que ya se ha comentado, el AS permite el acercamiento de los/as estudiantes a la comunidad, de modo de favorecer una oportunidad de poner en práctica sus conocimientos (Facultad de Ciencias Físicas y Matemáticas, Universidad de Chile, 2019). Sin embargo, las propuestas de AS que implementan las universidades deben integrarse en el conjunto de cambios orientados hacia un nuevo modelo de universidad centrado en la responsabilidad social (Cleeland, 2013). En este sentido, la Universidad Católica de Temuco desde el año 2009 instala un Modelo Educativo y uno de sus ejes es la Formación Humanista 
Cristiana que busca fortalecer la formación integral de cada uno de sus estudiantes (Universidad Católica de Temuco, 2009).

En el marco de este modelo educativo, una de las iniciativas claves es el Programa de Formación para el Servicio Socio Comunitario que contiene la estrategia metodológica de Aprendizaje Servicio (AS) (Universidad Católica de Temuco, Dirección General de Docencia, 2016). Desde el año 2012, la Dirección de Formación Humanista Cristiana (DFHC) ha desarrollado un sistema de gestión para la implementación de AS en cada carrera y, de modo escalonado, ha incorporado en cada plan de estudios esta estrategia para el aprendizaje. En esta primera etapa se han diagnosticado algunas dificultades tales como: tiempo de dedicación para la implementación, necesidad de capacitación, asesoramiento de equipos especializados y acompañamiento a docentes y a cursos.

Finalmente, la instalación de AS en la Universidad se declara y sustenta en la Resolución de Vicerrectoría Académica Nº6/2017 (Universidad Católica de Temuco, 2017), documento que reformaliza la renovación de la Formación Humanista Cristiana en la Universidad, promoviendo el AS como una estrategia de integración de competencias genéricas identitarias para el aprendizaje significativo de los/as estudiantes (Universidad Católica de Temuco, 2016).

En esta universidad, la implementación del modelo de AS ha considerado un proceso de cinco fases: Comisión de Trabajo, Análisis curricular, Experiencia piloto, Rediseño y Consolidación (Universidad Católica de Temuco, Dirección General de Docencia, 2016). La primera etapa tiene relación con generar la conformación de un equipo profesional que cautele la implementación de AS, dentro del itinerario formativo de una carrera. La segunda, conlleva el análisis curricular, la pertinencia de implementar AS, la capacitación de los docentes (equipo de trabajo), establecer vínculos con socios comunitarios y el diseño de la actividad piloto. En la tercera fase de la realización de talleres a los/as estudiantes sobre AS, se implementa la actividad, así como la evaluación del proceso. La cuarta fase requiere la revisión de los resultados de la actividad de AS, el rediseño de la guía de aprendizaje, reiterar la experiencia retroalimentada y su seguimiento. La última fase comprende una evaluación sistemática de las actividades realizadas, sus alcances, así como la difusión de los resultados (Fig. 1).

Ahora bien, la Universidad ha definido la incorporación de cuatro criterios de selección de cursos en el Itinerario formativo de las carreras técnicas y profesionales para la incorporación de AS. Estos criterios permiten el análisis del itinerario formativo (malla) y la selección de acciones curriculares (cursos) donde poder fijar el AS, eso sí, respetando la naturaleza y originalidad de cada disciplina y el proceso histórico de cada carrera. Los criterios definidos son los siguientes: representativo de la carrera, fortalecimiento a la identidad profesional, movilización de competencias genéricas identitarias, potenciador del vínculo focalizado de la Universidad con el medio para poder asegurar la permanencia en el tiempo y, de ese modo, ser un aporte más concreto a la transformación social a través de una necesidad abordada 


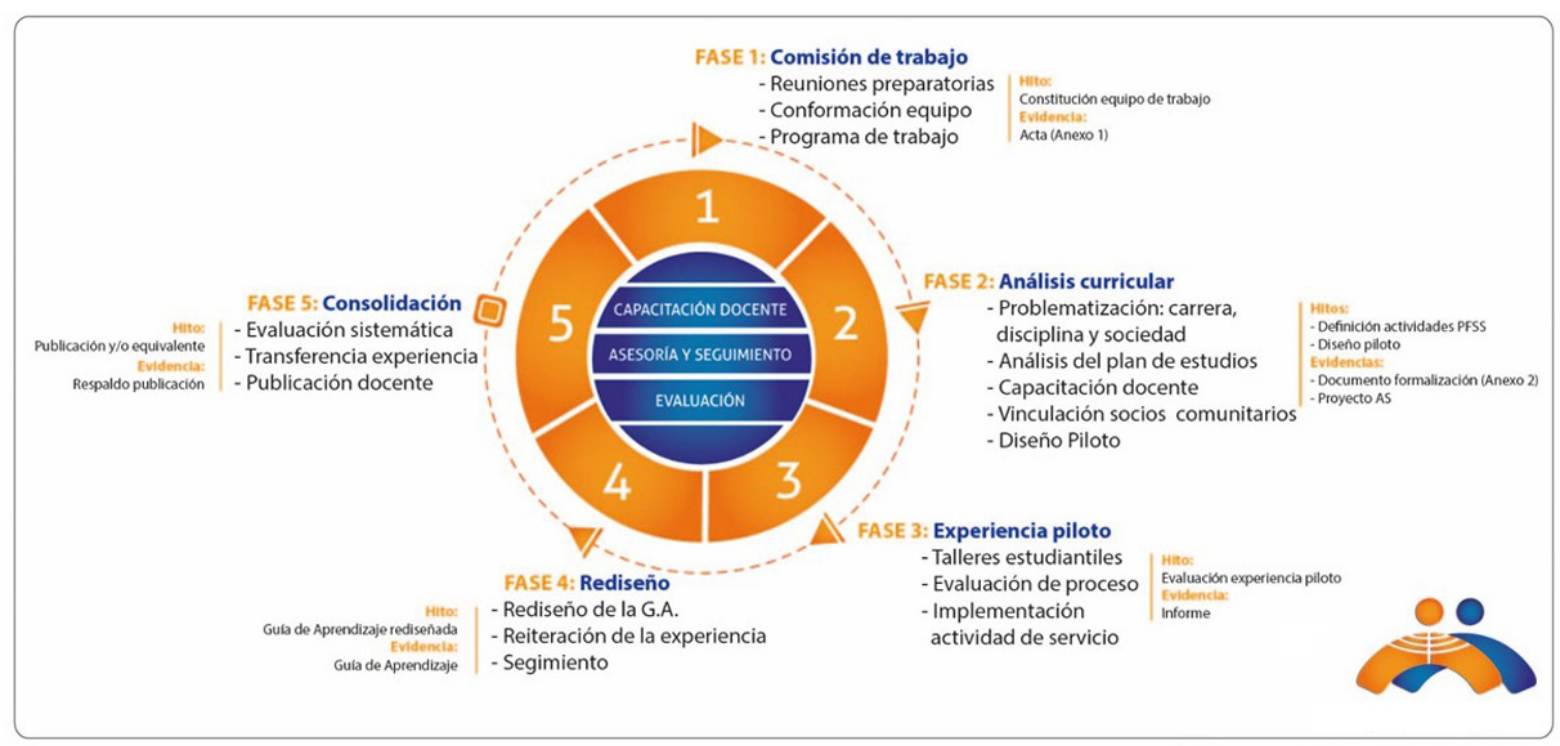

Fig. 1. Fases del proceso de instalación de AS en la Universidad Católica de Temuco.

Fuente: Dirección General de Docencia, Dirección de Formación Humanista Cristiana, Vicerrectoría Académica Universidad Católica de Temuco.

(Garbarini, 2013). No obstante lo anterior, estos criterios deben ser considerados como un eje crítico, el cual operativamente puede relacionarnos con el currículo general de la carrera en vínculo con su respectiva facultad.

Cabe destacar finalmente que una particularidad del proceso de instauración del MAS en esta universidad considera la formación de ayudantes especializados, para que apoyen las actividades de Aprendizaje Servicio en los cursos y, en especial, al acompañamiento entre pares para colaborar al logro de los resultados de aprendizaje.

\section{ALGUNAS EXPERIENCIAS DE APRENDIZAJE SERVICIO EN LA UC TEMUCO}

En coherencia con el proyecto educativo y con el Modelo Aprendizaje Servicio, la Universidad Católica de Temuco ha realizado esfuerzos por integrar a las distintas carreras en el desarrollo de actividades socio-educativas enmarcadas en esta perspectiva. En tal sentido, se puede ver en el cuadro que se presenta más adelante, que una importante cantidad de carreras y experiencias se han desarrollado. Éstas no están ordenadas por año, dado que en algunos casos se han ido desarrollando sistemáticamente desde que se implementa el Modelo Aprendizaje Servicio. Lo que se puede apreciar es que hay una tendencia a trabajar en áreas de infancia, adultos mayores y temáticas relacionadas con el desarrollo de la comunidad (ver Tabla 1).

Se puede apreciar en este cuadro que son distintas las disciplinas que se han incorporado a este tipo de experiencias. Sin embargo, no se cuenta con una sistematización evaluativa por parte de las carreras en relación a estos procesos que permita hacer un análisis en relación al aprendizaje significativo de las/os estudiantes, los aportes a la comunidad, las instituciones 


\section{VIVERO et al.}

Tabla 1. Descripción experiencias AS Universidad Católica de Temuco.

\begin{tabular}{cccl}
\hline Carrera & Asignatura & Institución/Organización & \multicolumn{1}{c}{ Actividad AS } \\
\hline Agronomía & Producción Hortícola & $\begin{array}{l}\text { Ministerio de Desarrollo } \\
\text { Social, programa } \\
\text { autoconsumo. }\end{array}$ & $\begin{array}{l}\text { Apoyo en realización de huertos } \\
\text { ecológicos. 15 familias del programa } \\
\text { autoconsumo del ministerio }\end{array}$ \\
\hline Antropología & Antropología Aplicada & Municipalidad de Saavedra & $\begin{array}{l}\text { Estudio colaborativo en } \\
\text { levantamiento de información, } \\
\text { sistematización y generación de } \\
\text { propuestas para la Mesa Indígena y } \\
\text { Municipalidad de Saavedra }\end{array}$ \\
\hline
\end{tabular}

$\begin{array}{lll}\text { Contador } & \text { Taller de Consultoría } & \begin{array}{l}\text { Municipalidad de Nueva } \\ \text { Amperial }\end{array}\end{array}$

Asesoría a microempresarios de diferentes rubros económicos de la comuna de Nueva Imperial

\begin{tabular}{llll} 
Educación de & Lenguajes Artísticos: & Jardines Infantiles y & “Teatro Infantil” dirigido a niñas y \\
Párvulos & Arte y Literatura & Temuco de la Comuna de & $\begin{array}{l}\text { niños de los Jardines Infantiles y } \\
\text { Escuelas de Enseñanza Básica }\end{array}$ \\
\hline
\end{tabular}

\begin{tabular}{lll}
$\begin{array}{l}\text { Escuela de } \\
\text { Ciencias de la } \\
\text { Salud }\end{array}$ & $\begin{array}{l}\text { Módulos Integrados en } \\
\text { Salud II }\end{array}$ & $\begin{array}{l}\text { Escuelas, Colegios y Liceos } \\
\text { de la Región de la Araucanía }\end{array}$ \\
\hline Geografía & $\begin{array}{l}\text { Desarrollo Local y } \\
\text { Regional / Geografía } \\
\text { Rural }\end{array}$ & Municipalidad de Lonquimay
\end{tabular}

Diagnósticos participativos

Diagnóstico territorial participativo en la comuna de Lonquimay. Reflexiones respecto a su situación actual y futura

Ingeniería Civil Taller de Integración II en Informática Taller de Integración IV $\begin{array}{ll}\text { Colegio de Ayudantes UCT } & \begin{array}{l}\text { Plataforma de aprendizaje entre } \\ \text { pares }\end{array}\end{array}$

\begin{abstract}
Medicina
Veterinaria

Diagnóstico Semiológico

\section{Educación Liderazgo y Trabajo \\ Diferencial Colaborativo}

Municipalidad de Padre Las

Casas. Pequeños agricultores
Consultorio de Caballos Carretoneros
Centros de Practica adscritos al Taller Pedagógico Nivel III. Escuelas que cuentan con proyectos de Integración. Comuna de Temuco
Diagnóstico de necesidades educativas y situaciones de exclusión en el contexto en el cual se desarrolla la práctica pedagógica
Pedagogía En Educación Física
Desarrollo y Evaluación Motriz Aplicada
Jardines infantiles de la ciudad de Temuco
Desarrollo de habilidades motoras básicas de los niños y niñas 


\section{CONCLUSIONES}

La implementación del neoliberalismo en Chile se materializa, entre otros hechos, en la mercantilización de uno de los bienes públicos claves para el desarrollo social inclusivo, como lo es el derecho a la educación (Atria, 2013, 2014; Bellei, 2015; Brown, 2015; Gaudichaud, 2015). Como es sabido, a principios de la década del ochenta del siglo XX, se realizaron diversas "reformas estructurales" de corte neoliberal, las que fueron implementadas en el contexto de una dictadura militar (Moulian, 1997, 2009; Brown, 2015; Gaudichaud, 2015) y sus efectos se expresan contemporáneamente en la configuración de ciertas condiciones materiales y subjetivas en el campo de la educación chilena actual. Por ello, nos resulta preocupante que el MAS se implemente desde una supuesta neutralidad axiológica y política, que no hace sino encubrir las causas estructurales de las desigualdades e inequidades educativas en el acceso, permanencia y egreso en la educación superior.

Frente al escenario de convulsión social, que pone en evidencia la crisis de legitimidad del neoliberal y las relaciones sociales mercantilizadas, nos parece fundamental que el eje central de cualquier intervención en el campo del aprendizaje parta del análisis y reflexión crítica del contexto histórico-político. Por lo tanto, resulta necesario se problematice la implementación de estos modelos y no se caiga en una complacencia ingenua y acrítica en su implementación. Es decir, no puede seguir siendo guiada por la racionalidad instrumental y por la "razón neoliberal" (Brown, 2015), como ha sido hasta ahora su puesta en práctica en el campo de la educación universitaria en Chile.

El MAS, generalmente, se ha presentado como una metodología de enseñanzaaprendizaje innovadora, que demanda que el docente posea una mirada globalizante frente a nuevas formas de hacer docencia universitaria y adquiera compromisos institucionales. En el despliegue de AS en contexto universitario, se observa una tendencia a no problematizar una dimensión clave que es inherente al modelo. Nos referimos al análisis crítico del contexto político-social, económico y cultural, donde los actores universitarios materializan un servicio profesional o disciplinario que aspira a generar un impacto comunitario o local, en los cuales se insertan determinadas Instituciones de Educación Superior (IES).

Que las/os estudiantes, como quienes asumen como guías del proceso académicopráctico, se sitúen desde el análisis histórico-político, es decir, una toma de conciencia de la realidad material permitirá reconocer hasta dónde las actividades de AS permiten generar aporte significativo para los agentes sociales o comunitarios con quienes actúan. Implica darle un sentido valórico, ético y cultural, que permita, por un lado, ir generando vínculos comunitarios y, por otro, el fortalecimiento de la identidad profesional. Ello contribuiría a que las/os estudiantes se sientan comprometidos socialmente y profesionales con un sello distintivo de determinadas IES que despliegan sus actividades en contextos comunitarios con características locales específicas. 
El escenario actual deja en capilla a todos los modelos que, de una u otra forma, han sido tributarios del neoliberalismo. El MAS, sin duda, debe ser problematizado críticamente si se quiere contribuir a la necesaria transformación social, donde las/os estudiantes y la academia en general puedan ser parte activa de este nuevo Chile. Así entonces, el aprendizaje servicio también debe mirarse como una oportunidad para repensar la relación que ha tenido la academia con sus contextos sociales más próximos, no como una vinculación mesiánica, sino como parte de actores sociales que conjunta y dialécticamente van transformando y transformándose en un contexto histórico-social y político concreto. La exploración del modelo implementado por la Universidad Católica de Temuco sobre la incorporación de la estrategia innovadora de Aprendizaje Servicio (AS), por sí misma no explica su aporte al desarrollo social, sino que ello será posible de determinar en la medida que las relaciones con los actores sociales comunitarios se generen de manera más horizontal, menos mesiánica, y sobre la base de un compromiso ético y político de mejorar las condiciones de vida de los sectores más excluidos.

\section{REFERENCIAS}

Aguilar, M., \& Bize, R. (2000). Pedagogía de la Intencionalidad. Rosario: Homo Sapiens.

Arratia, A. (2008). Ética, Solidaridad y Aprendizaje Servicio en la Educación Superior. Acra bioethica, 14(1), 61-67.

Asún, R., Zúñiga, C., \& Ayala, Ma. (2013). La formación por competencias y los Estudiantes: confluencias y divergencias. En la construcción del docente ideal. Calidad en la educación, 38, 277-304.

Atria, F. (2013). Veinte años después Neoliberalismo con rostro humano. Santiago: Editorial Catalonia.

Atria, F. (2014). Derechos sociales y educación: Un nuevo paradigma de lo público. Santiago de Chile: Editorial Lom.

Barrios Araya, S., Rubio Acuña, M., Gutiérrez Núñez, M., \& Sepúlveda Veria, C. (2012). Aprendizaje-servicio como metodología para el desarrollo del pensamiento crítico en educación superior. Educación Media Superior, 26(24), 594-603.

Bellei, C. (2015). El gran experimento. Mercado y privatización de la educación chilena. Santiago de Chile: Editorial Lom.

Brown, W. (2015). El pueblo sin atributos. La secreta revolución del neoliberalismo. Barcelona: Editorial Malpaso.

Canales, M. (2006). Metodologías de investigación social. Introducción a los oficios. Santiago de Chile: Editorial Lom.

Canales, M. (2013a). Escucha la escucha. Análisis e interpretación en la investigación cualitativa. Santiago de Chile: Editorial Lom.

Canales, M. (2013b). Investigación social. Lenguaje del diseño. Santiago de Chile: Editorial Lom. 
Centro Latinoamericano de Aprendizaje y Servicio Solidario. (11 de abril de 2019). http:// creasfile.uahurtado.cl. Obtenido de http://creasfile.uahurtado.cl/estadodeAS. Enrique\%20Ochoa.pdf

Centro Promotor de Aprendizaje Servicio. (11 de abril de 2019). aprenentatgeservei.org. Obtenido de aprenentatgeservei.org: http://www.aprenentatgeservei.org/versions. php?l=18

Cleeland, M. (2013). Aprendizaje servicio desde el enfoque comunitario: un modelo de intervención social en la educación superior. Tesis. Santiago: Universidad de Chile.

Cooper, S., Cripps, J., \& Reisman, J. (2013). Service-Learning in Deaf Studies: Impact on the development of altruistic behaviors and social justice concern. American Annals of the deaf, 157(5), 413-427.

Espinoza, F., \& Rodríguez de la Piedra, V. (2015). Aprendizaje Servicio, una estrategia de aprendizaje significativo en la formación de estudiantes de Terapia Ocupacional de la Universidad de Chile. Revista Chilena de Terapia Ocupacional, 15, 11-18.

Eyler, J. (2001). Creating your reflection map. New directions for higher education. Creating your reflection map. New directions for higher education, 114, 35-42.

Facultad de Ciencias Físicas y Matemáticas, Universidad de Chile. (13 de abril de 2019). ingenieria.uchile.cl. Obtenido de ingenieria.uchile.cl: http://ingenieria.uchile.cl/ extension/extension-en-la-fcfm/132012/aprendizaje-y-servicio-as

Freire, P. (1996). Política y educación. Buenos Aires: Editorial Siglo XXI.

Freire, P. (2004). La educación como práctica de la libertad. Buenos Aires: Editorial Siglo XXI. Freire, P. (2006). Pedagogía del Oprimido. Buenos Aires: Editorial Siglo XXI.

Garbarini, C. (05 de mayo de 2013). Criterios de selección de cursos para AS. (entrevista de Danitza Standen Silva).

Gaudichaud, F. (2015). Las fisuras del neoliberalismo chileno. Trabajo, crisis de la "democracia tutelada" y conflictos de clase. Santiago de Chile: Quimantú y Tiempo Robado.

Hansen, K. (2012). A practical guide for designing a course with a Service-Learning component in higher education. The Journal of Faculty Development, 26(1), 29-36.

Jouannet, C., Salas, M., \& Contreras, M. A. (2013). Modelo de implementación de Aprendizaje Servicio $(\mathrm{A}+\mathrm{S})$ en la UC: Una experiencia que impacta positivamente en la formación profesional integral. Calidad en la educación, 39, 197-212.

Lepeley, M. T. (2001). Gestión y calidad en educación: un modelo de evaluación. Santiago: McGraw-Hill Interamericana.

Morin, E. (2006). El método 6. Ética. Madrid: Cátedra.

Moulian, T. (1997). Chile actual: anatomía de un mito. Santiago de Chile: Editorial Lom

Moulian, T. (2009). Contradicciones del desarrollo político chileno. 1920-1990. Santiago de Chile: Editorial Lom.

Puig Rovira, J., Girón Casares, M., Martín García, X., \& Rubio Serrano, L. (2011). Aprendizaje-servicio y Educación para la Ciudadanía. Revista de Educación, Número extraordinario, 45-67. 
Red de Aprendizaje y Servicio, Chile. (11 de abril de 2019). REASE: red de Aprendizaje y Servicio, Chile. Obtenido de REASE: red de Aprendizaje y Servicio, Chile: https:// reasechile.wordpress.com/

Santander, G. (2017). Aprendizaje servicio como herramienta metodológica en la educación superior. Revista Iberoamericana de Aprendizaje Servicio, 3, 159-163.

Tapia, M. N. (2008). Calidad académica y responsabilidad social: el aprendizaje servicio como puente entre dos culturas universitarias. En X. Martínez (Ed.), Aprendizaje servicio y responsabilidad social en las universidades (pp. 27-56). Barcelona: Ediciones Octaedro.

Tobón, S. (2008). La formación basada en competencias en la educación superior: El enfoque complejo. Universidad autónoma de guadalajara. Curso iglu 2008 guadalajara. México. Bogotá: Instituto Cife.ws.

Ugueño, A. (2015). Aprendizaje servicio: Un espacio de formación práctica que valida el conocimiento del otro y la reciprocidad de saberes. Experiencias desde la Universidad Católica de Temuco, Chile. En L. E. González (Ed.), En varios autores/as: La formación práctica en la universidad y su impacto en el perfil de egreso. Editado por el Centro Interuniversitario de Desarrollo (CINDA) (pp. 245-258). Santiago de Chile: CINDA.

Unesco. (1997). La educación encierra un Tesoro. Unesco. París: Santillana, Ediciones Unesco. Obtenido de http://www.unesco.org/education/pdf/DELORS_S.PDF

Universidad Católica de Temuco. (2009). Modelo Educativo de UC Temuco, Principios y Lineamientos. Temuco: Dirección General de Docencia. UCTemuco.

Universidad Católica de Temuco.(2016). Competencias Genéricas para la Formación deProfesionales integrales. Temuco: Dirección General de Docencia. Cuaderno de Docencia N 3.

Universidad Católica de Temuco. (2016). Plan de Desarrollo Institucional 2010-2020, Periodo de Gestión 2016-2020. Temuco: Dirección General de Gestión Institucional.

Universidad Católica de Temuco. (2017). Resolución de Vicerrectoría Académica. Formaliza la renovación de la formación humanista cristiana. Temuco: Vicerrectoría Académica.

Universidad Católica de Temuco. (2019). Informe de autoevaluación Institucional, proceso de acreditación 2019. Temuco: Universidad Católica de Temuco.

Universidad Católica de Temuco, Dirección General de Docencia. (2016). Programa de Formación para el Servicio Socio comunitario. Temuco: Dirección General de Docencia, UCTemuco.

Van Dijk, T. (1989). La ciencia del texto. Barcelona: Paidós.

Van Dijk, T. (1996). Estructuras y funciones del discurso. Una introducción interdisciplinaria a la lingüística y a los estudios del discurso. Ciudad de México D.F.: Siglo XXI.

Villa, A., \& Poblete, M. (2007).Aprendizaje basado en competencias. Una propuesta para la evaluación de las competencias genéricas. Bilbao: Universidad de Deusto. Ediciones Mensajero.

White, S., \& Henry, J. (1999). Incorporation of service-learning into a baccalaureate nursing education curriculum. Nurse Outlook, 47(6), 257-261. 